Article

\title{
Design and Investigation of a Dual Material Gate Arsenic Alloy Heterostructure Junctionless TFET with a Lightly Doped Source
}

\author{
Haiwu Xie ${ }^{\mathbb{D}}$, Hongxia Liu *(D), Shupeng Chen *, Tao Han and Shulong Wang \\ Key Laboratory for Wide-Band Gap Semiconductor Materials and Devices of Education, School of \\ Microelectronics, Xidian University, Xi'an 710071, China; xiehaiwu.love@163.com (H.X.); \\ taohan373@gmail.com (T.H.); slwang@xidian.edu.cn (S.W.) \\ * Correspondence: hxliu@mail.xidian.edu.cn (H.L.); spchen@xidian.edu.cn (S.C.); \\ Tel.: +86-130-8756-8718 (H.L.); +86-189-9123-3677 (S.C.)
}

Received: 9 September 2019; Accepted: 26 September 2019; Published: 1 October 2019

\begin{abstract}
This paper designs and investigates a novel structure of dual material gate-engineered heterostructure junctionless tunnel field-effect transistor (DMGE-HJLTFET) with a lightly doped source. Similar to the conventional HJLTFET, the proposed structure still adopts an InAs/GaAs $\mathrm{As}_{0.1} \mathrm{Sb}_{0.9}$ heterojunction at source and channel interface and employs a polarization electric field at the arsenic heterojunction induced by the lattice mismatch in the InAs and $\mathrm{GaAs}_{0.1} \mathrm{Sb}_{0.9}$ zinc blende crystal to improve band to band tunneling (BTBT) current. However, the gate electrode is divided into three parts in DMGE-HJLTFET namely the auxiliary gate (M1), control gate (M2) and tunnel gate (M3) with workfunctions $\Phi_{\mathrm{M} 1}, \Phi_{\mathrm{M} 2}$ and $\Phi_{\mathrm{M} 3}$, where $\Phi_{\mathrm{M} 1}=\Phi_{\mathrm{M} 3}<\Phi_{\mathrm{M} 2}$, which not only improves ON-state current but also decreases the OFF-state current. In addition, a lightly doped source is used to further decrease the OFF-state current of this device. Simulation results indicate that DMGE-HJLTFET provides superior metrics in terms of logic and analog/radio frequency (RF) performance as compared with conventional HJLTFET, the maximum ON-state current and transconductance of the DMGE-HJLTFET increases up to $5.46 \times 10^{-4} \mathrm{~A} / \mu \mathrm{m}$ and $1.51 \times 10^{-3} \mathrm{~S} / \mu \mathrm{m}$ at $1.0 \mathrm{~V}$ drain-to-source voltage (Vds). Moreover, average subthreshold swing (SSave) of DMGE-HJLTFET is as low as $15.4 \mathrm{mV} / \mathrm{Dec}$ at low drain voltages. Also, DMGE-HJLTFET could achieve a maximum cut-off frequency $\left(f_{T}\right)$ of $423 \mathrm{GHz}$ at $0.92 \mathrm{~V}$ gate-to-source voltage (Vgs) and a maximum gain bandwidth (GBW) of $82 \mathrm{GHz}$ at Vgs $=0.88 \mathrm{~V}$, respectively. Therefore, it has great potential in future ultra-low power integrated circuit applications.
\end{abstract}

Keywords: lightly doped source; band to band tunneling (BTBT); arsenic alloy heterostructure; dual material gate-engineered heterostructure junctionless TFET(DMGE-HJLTFET)

\section{Introduction}

Advancement in the semiconductor industry has increased the demand for nanoscale devices for analog/ radio frequency (RF) and high switching speed applications, but, power consumption and reliability issues are the major concerns in integrated circuits (ICs), so compactness in devices is an urgent problem to be solved in nanoscale systems. According to Moore's law, drastic reduction in volume and cost of the devices will occur. However, as the conventional metal oxide semiconductor field effect transistors (MOSFETs) scale down continuously, the OFF-state current dramatically increases and short channel effects (SCE) are severely aggravated, and a limitation of $60 \mathrm{mV} / \mathrm{Dec}$ subthreshold swing (SS) cannot be broken. In addition, low switch ratio (Ion/Ioff) ratio and drain induced barrier lowering (DIBL) effect are also arising. In order to avoid these issues, a lot of novel structures have 
been proposed and investigated in recent years, among them, tunnel field effect transistor (TFET) [1,2] is a selective candidate for future ultra-low power applications, where carrier injection from source to channel is modulated by a gate-controlled band to band tunneling (BTBT) mechanism. The structure of a conventional TFET consists of a gated $\mathrm{p}$ type region/ intrinsic region $/ \mathrm{n}$ type region $(\mathrm{p}-\mathrm{i}-\mathrm{n})$ diode operating under a reverse bias condition and the gate bias determines the quantum tunneling barrier width at the source-channel interface. Benefitting from innovation of structure and mechanism, TFETs are compatible with conventional complementary metal oxide semiconductor (CMOS) process and can break the SS limit of $60 \mathrm{mV} / \mathrm{Dec}$. Additionally, it is not influenced by the short channel effects (SCE) and the leakage current of conventional TFETs is small under the OFF-state condition $[3,4]$. However, small ON-state current and large Miller capacitance are still inherent disadvantages in TFETs. To improve the ON-state current, a lot of novel structures of TFETs are proposed, for example, the L-shaped channel TFET (LTFET) [5-7], U-shaped channel TFET (UTFET) [8,9], symmetric TFET (S-TFET) [10,11], U-shaped channel with dual sources TFET (DS-UTFET) [12], covered source-channel TFETs (CSC-TFETs) [13], hetero junction TFET with T-shaped gate (HTG-TFET) [14], 2D materials channel TFET and hetero-bilayer TFETs $[15,16]$. As a whole, TFETs reported in recent years adopt abrupt junction at tunneling interface, which leads to a complex fabrication processes and a high thermal budget. In addition, a heavily doped concentration in the channel and active region are also challenging during the fabrication process and it is easy to be influenced by random dopant fluctuations (RDFs) [17-21].

To avoid complex fabrication processes and high thermal budgets in TFETs, the junctionless tunneling field effect transistor (JLTFET) [22-30] has been studied extensively in recent years, which uses uniformly high-doping concentration in the source, channel and drain regions so that the doping concentration and type of channel region are consistent with source region and drain region. Due to uniform doping, the JLTFET is immune to random dopant fluctuations (RDFs) and overcomes complex fabrication processes in manufacturing, meanwhile, source and drain region are formed by the charge plasma concept which further avoids high thermal budgets in the JLTFET. Whereas, low ON-state current still remains the most serious problem in junctionless TFETs due to the presence of barrier between source and channel.

In this paper, a novel structure of dual material gate-engineered heterostructure junctionless tunnel field-effect transistor (DMGE-HJLTFET) with a lightly doped source was proposed to improve the performance of a conventional HJLTFET [31,32], where the corresponding heavily $p$ type doping region / intrinsic region / heavily $\mathrm{n}$ type doping region $\left(\mathrm{P}^{+}-\mathrm{I}-\mathrm{N}^{+}\right)$structure can be realized by the charge plasma concept with appropriate workfunction for the polar gate (PG) and control gate (CG). The PG is located at the source region and has a larger work function than $\mathrm{CG}$ for inducing a $\mathrm{P}^{+}$source while CG is located at the middle for inducing the intrinsic channel. We used a InAs/ $\mathrm{GaAs}_{0.1} \mathrm{Sb}_{0.9}$ heterostructure at the source-channel interface to improve band to band tunneling (BTBT) current through the heterostructure of $\mathrm{InAs} / \mathrm{GaAs}_{0.1} \mathrm{Sb}_{0.9}$ and a polarization electric field at the arsenic heterojunction induced by the lattice mismatch in the arsenic alloy zinc blende crystal. Moreover, the gate electrode was divided into three parts namely auxiliary gate (M1), control gate (M2) and tunnel gate (M3) with workfunctions $\Phi_{\mathrm{M} 1}, \Phi_{\mathrm{M} 2}$ and $\Phi_{\mathrm{M} 3}$, respectively, where $\Phi_{\mathrm{M} 1}=\Phi_{\mathrm{M} 3}<\Phi_{\mathrm{M} 2}$. Appropriate workfunctions for the gate electrodes can further improve ON-state current and suppress OFF-state current. Consequently, the DMGE-HJLTFET provides superior metrics in terms of logic and analog/RF performance as compared with conventional a HJLTFET, that is to say, it can generate higher ON-state current, higher transconductance, higher output transconductance and higher $f_{\mathrm{T}}$ and GBW. Therefore, it will have great potential in future ultra-low power and high frequency integrated circuit applications [33-37].

The paper is organized as follows: Section 2 describes the initial device parameters and essential simulation models. Section 3 introduces the properties of the structure and the optimization process. Section 4 shows the conclusions. 


\section{Methods}

Figure 1a shows the device structure of a conventional HJLTFET, in which the PG is located at the source region and has a larger workfunction than CG for inducing a $\mathrm{P}^{+}$source while $\mathrm{CG}$ is located at the middle for inducing an intrinsic channel, InAs/GaAs $\mathrm{Gs}_{0.1} \mathrm{Sb}_{0.9}$ heterostructure is introduced at the source-channel interface and a heavy doping is considered in the silicon body with concentration of $1 \times 10^{19} \mathrm{~cm}^{-3}$, resulting in a junctionless $\mathrm{P}^{+}-\mathrm{I}-\mathrm{N}^{+}$structure with appropriate workfunctions for PG and CG by the charge plasma concept. Compared with a conventional HJLTFET, a DMGE-HJLTFET still uses InAs/GaAs $\mathrm{Ga}_{0.1} \mathrm{Sb}_{0.9}$ at the source-channel interface and introduces a lightly doped source, moreover, the gate electrode is divided into three parts namely auxiliary gate (M1), control gate (M2) and tunnel gate (M3) with workfunctions $\Phi_{\mathrm{M} 1}, \Phi_{\mathrm{M} 2}$ and $\Phi_{\mathrm{M} 3}$, respectively, where $\Phi_{\mathrm{M} 1}=\Phi_{\mathrm{M} 3}<\Phi_{\mathrm{M} 2}$, as shown in Figure $1 \mathrm{~b}$. As a result, the DMGE-HJLTFET has larger ON-state current and lower OFF-state current than HJLTFET, so it will have great potential to be applied in future ultra-low power integrated circuit.

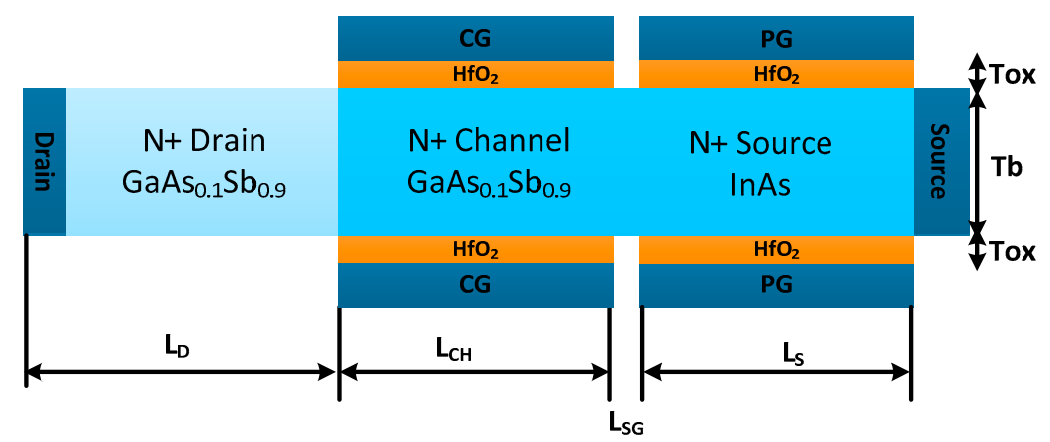

a

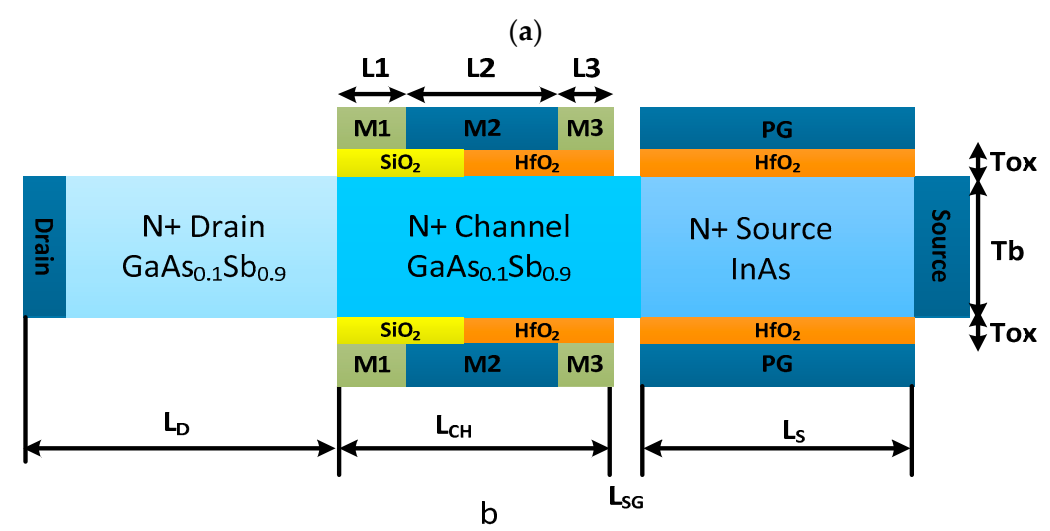

(b)

Figure 1. Cross-sectional view of (a) a conventional heterostructure junctionless tunnel field-effect transistor (HJLTFET); (b) a dual material gate-engineered (DMGE)-HJLTFET.

The detailed parameters of the conventional HJLTFET and DMGE-HJLTFET used in the simulation were as follows: the length of source region $\left(\mathrm{L}_{S}\right)$, the length of channel region $\left(\mathrm{L}_{\mathrm{CH}}\right)$ and the length of drain region $\left(L_{D}\right)$ were all $20 \mathrm{~nm}$; the gap length $\left(L_{S G}\right)$ between source and channel was $5 \mathrm{~nm}$; the thickness of $\mathrm{HfO}_{2}\left(\mathrm{~T}_{\mathrm{OX}}\right)$ was $2 \mathrm{~nm}$, the thickness of the body $(\mathrm{Tb})$ was $5 \mathrm{~nm}$; the length of auxiliary gate (L1) was $7 \mathrm{~nm}$, the length of control gate (L2) was $10 \mathrm{~nm}$, the length of tunnel gate (L3) was $3 \mathrm{~nm}$; the workfunction of polarity gate $\left(\Phi_{\mathrm{PG}}\right)$ was $5.9 \mathrm{eV}$, the workfunction of control gate $\left(\Phi_{\mathrm{CG}}\right)$ was $4.4 \mathrm{eV}$, the workfunction of auxiliary gate $\left(\Phi_{\mathrm{M} 1}\right)$ and the work function of tunnel gate $\left(\Phi_{\mathrm{M} 3}\right)$ were $4.1 \mathrm{eV}$. In addition, the N-type doping concentration of the channel region and drain region were all $1 \times 10^{19} \mathrm{~cm}^{-3}$ in the HJLTFET and DMGE-HJLTFET; whereas the N-type doping concentration of source region in the HJLTFET and DMGE-HJLTFET were $1 \times 10^{19} \mathrm{~cm}^{-3}$ and $5 \times 10^{17} \mathrm{~cm}^{-3}$, respectively. 
All the simulations were performed using ATLAS Silvaco TCAD version 5.20.2.R (Silvaco International, Santa Clara, CA, USA). To consider the band to band tunneling phenomenon in the case of TFET and the spatial variation of the energy bands, the nonlocal BTBT model (BBT.NONLOCAL) was used. The presence of a highly doped channel meant that the Shockley-Read-Hall related to concentration (CONSRH) was activated to account for the minority carrier recombination effects. In addition to this, Fermi statistics (FERMI) and band gap narrowing (BGN) model were also activated. Moreover, a quantum confinement model given by Hansch (HANSCHQM) was also used to take into account quantum confinement effects due to the increased doping levels and thinner gate oxide in the channel. Furthermore, the Schenk model for trap-assisted tunneling (SCHENK.TUNN) was also involved to include the tunneling of electrons from the valence band to the conduction band through trap or defect states and phonon-assisted tunneling effects.

\section{Results and Discussion}

3.1. The Physical Mechanism of dual material gate-engineered heterostructure junctionless tunnel field-effect transistor (DMGE-HJLTFET)

The physical mechanism of the DMGE-HJLTFET is shown in Figure 2a-d. Figure 2a shows the nonlocal BTBT tunneling rate of electrons and Figure $2 b$ shows the nonlocal BTBT tunneling rate of holes of the DMGE-HJLTFET when $\mathrm{Vds}=1 \mathrm{~V}$ and $\mathrm{Vgs}=1.2 \mathrm{~V}$. It was obvious to observe that BTBT mainly occurred at the source and channel interface, and the rate of BTBT increased significantly due to the heterojunction between source and channel. In Figure 2c, the electric field distribution of DMGE-HJLTFET at Vds $=1 \mathrm{~V}$ and $\mathrm{Vgs}=1.2 \mathrm{~V}$ is illustrated, it is not difficult to find from this figure that value of electric field was markedly improved near the hetero junction and hetero dielectric, so the ON-state current significantly increased. In addition, the total current density of DMGE-HJLTFET was researched in order to well understand the physical mechanism, as shown in Figure 2d.

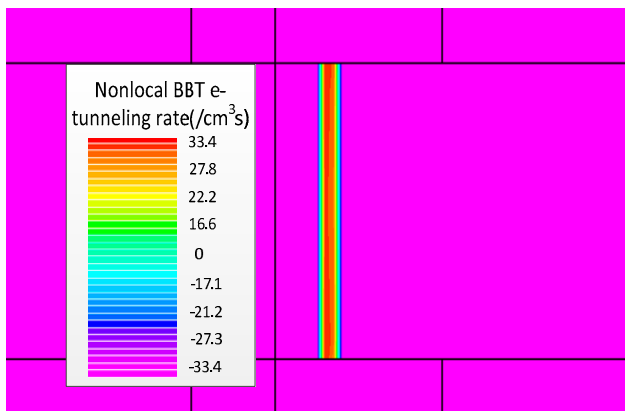

(a)

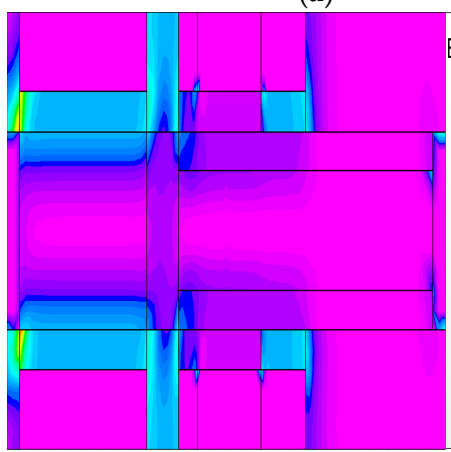

(c)

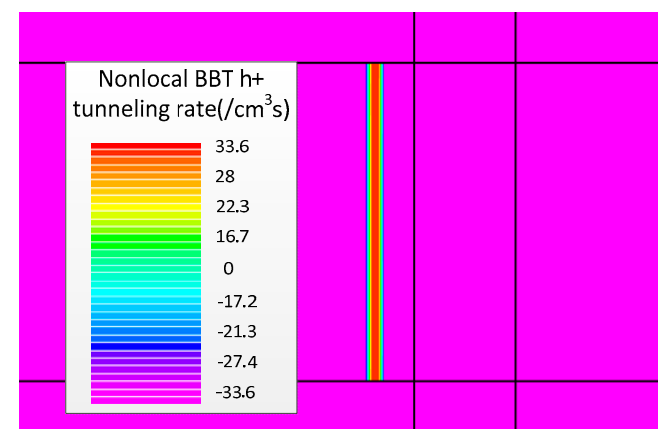

(b)

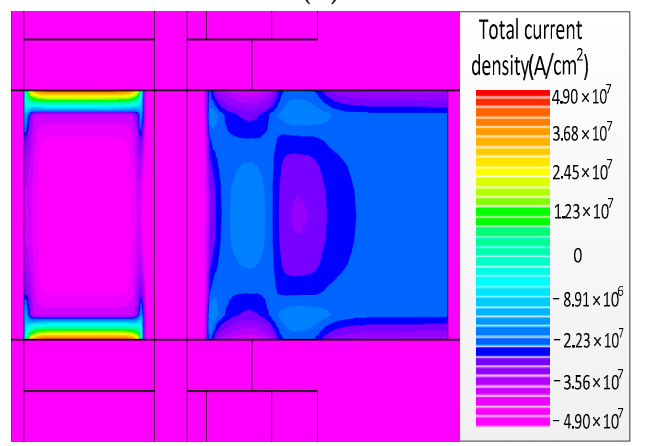

(d)

Figure 2. (a) Nonlocal band to band tunneling (BTBT) rate of electrons; (b) nonlocal band to band tunneling (BTBT) rate of holes; (c) electric field distribution in DMGE-HJLTFET; (d) total current density of DMGE-HJLTFET. 
Figure 3a shows the energy band diagram of the HJLTFET and DMGE-HJLTFET in ON-state $(\mathrm{Vds}=1 \mathrm{~V}, \mathrm{Vgs}=1 \mathrm{~V})$, where it was noticed that the conduction band and valance band of DMGE-HJLTFET at the source-channel interface were very close to each other and the tunneling distance of DMGE-HJLTFET was much smaller than the HJLTFET, i.e., tunneling width and effective tunneling area of DMGE-HJLTFET were obviously improved by appropriate workfunctions for the divided gate electrode as compared with HJLTFET, resulting in enhancement on electron probability to tunnel from the source to channel junction in the DMGE-HJLTFET. Figure $3 b$ indicates the energy band diagram of HJLTFET and DMGE-HJLTFET in OFF-state (Vds $=1 \mathrm{~V}, \mathrm{Vgs}=0 \mathrm{~V})$, and from this figure it is not difficult to find that the distance of conduction band and valance band in source/channel interface of the DMGE-HJLTFET was much larger than HJLTFET. In addition, an extra barrier height occurred in the channel region for DMGE-HJLTFET. This is because the gate electrode was divided into three parts in the DMGE-HJLTFET namely the auxiliary gate (M1), control gate (M2) and tunnel gate (M3), and the auxiliary gate (M1) produced the extra barrier height in OFF-state.

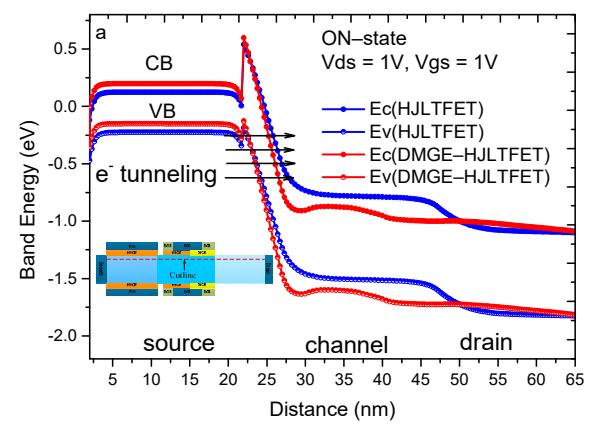

(a)

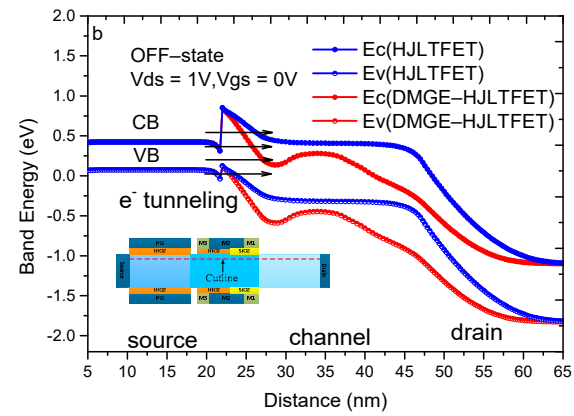

(b)

Figure 3. (a) The ON-state energy band diagram of the HJLTFET and DMGE-HJLTFET; (b) the OFF-state energy band diagram of the HJLTFET and DMGE-HJLTFET.

\subsection{The Input Characteristics}

Figure 4a illustrates the transfer characteristics of HJLTFET and DMGE-HJLTFET. As can be seen clearly from this figure that the DMGE-HJLTFET can produce higher ON-state current than HJLTFET, and the ON-state current of the DMGE-HJLTFET was $5.46 \times 10^{-4} \mathrm{~A} / \mu \mathrm{m}$ at $\mathrm{Vgs}=1.2 \mathrm{~V}$, however, the corresponding ON-state current of the HJLTFET was only $1.05 \times 10^{-4} \mathrm{~A} / \mu \mathrm{m}$. Meanwhile, the OFF-state current of DMGE-HJLTFET was $8.4 \times 10^{-18} \mathrm{~A} / \mu \mathrm{m}$ while the OFF-state current of HJLTFET was $3.1 \times 10^{-17} \mathrm{~A} / \mu \mathrm{m}$. Consequently, compared with the conventional HJLTFET, the Ion/Ioff ratio of the DMGE-HJLTFET was increased by one order of magnitude. In addition, the DMGE-HJLTFET has a smaller average subthreshold swing (SSave) because of structural innovation, the SSave (which is extracted from Vmin to $\mathrm{Vt}$; Vmin is the gate voltage where drain current equals to Ioff, and the gate voltage where the drain current becomes $1 \times 10^{8} \mathrm{~A} / \mu \mathrm{m}$ is taken as the threshold voltage $\mathrm{Vt}$ ) values of the DMGE-HJLTFET and HJLTFET were $15.4 \mathrm{mV} / \mathrm{Dec}$ and $24.5 \mathrm{mV} / \mathrm{Dec}$, respectively.

The transconductance $\left(\mathrm{g}_{\mathrm{m}}\right)$ is an important parameter to evaluate the analog performance of devices, which is defined as the first derivative of drain current (Ids) with respect to $\mathrm{V}_{\mathrm{GS}}$, the formula of $g_{m}$ is given by Equation (1):

$$
\mathrm{g}_{\mathrm{m}}=\frac{\mathrm{dI}_{\mathrm{ds}}}{\mathrm{dV}_{\mathrm{gs}}} .
$$

As shown in Figure $4 \mathrm{~b}$, the $\mathrm{g}_{\mathrm{m}}$ of the DMGE-HJLTFET increased observably compared with HJLTFET. The maximum $g_{m}$ value of the DMGE-HJLTFET was $1.51 \times 10^{-3} \mathrm{~S} / \mu \mathrm{m}$ at $\mathrm{Vgs}=1.14 \mathrm{~V}$, while the maximum $g_{m}$ value of the HJLTFET was $6.02 \times 10^{-4} \mathrm{~S} / \mu \mathrm{m}$. Therefore, the maximum $\mathrm{g}_{\mathrm{m}}$ of the DMGE-HJLTFET was almost 26 times more than the HJLTFET. 


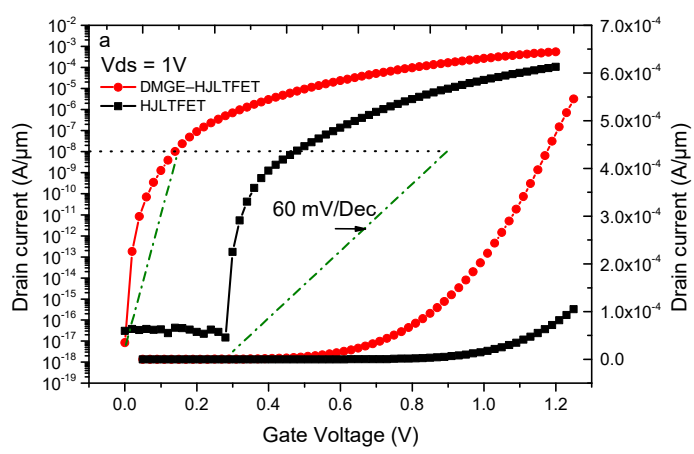

(a)

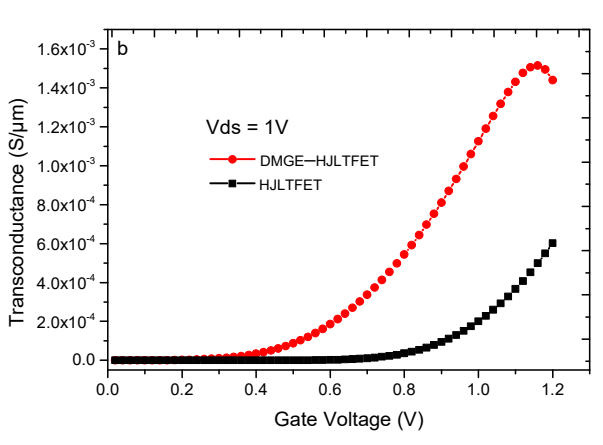

(b)

Figure 4. (a) The transfer characteristics of the HJLTFET and DMGE-HJLTFET; (b) transconductance of the HJLTFET and DMGE-HJLTFET.

\subsection{The Output Characteristics}

Figure 5a indicates the output characteristics of the HJLTFET and DMGE-HJLTFET at Vgs = $1 \mathrm{~V}$, it can be seen clearly that HJLTFET showed worse saturation performance when gate voltage was less than $0.5 \mathrm{~V}$, meanwhile, the maximum output saturation drain current of HJLTFET was only $2.35 \times 10^{-5} \mathrm{~A} / \mu \mathrm{m}$. While the DMGE-HJLTFET exhibited better saturation performance within the simulated voltage range, and the maximum output saturation drain current of DMGE-HJLTFET increased up to $2.66 \times 10^{-4} \mathrm{~A} / \mu \mathrm{m}$.

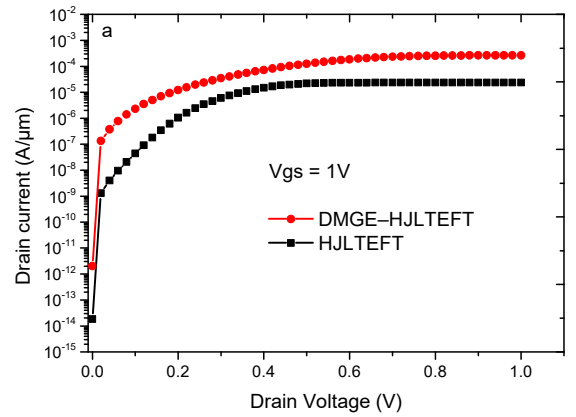

(a)

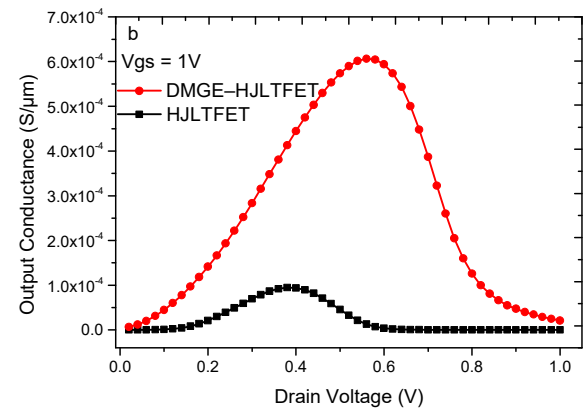

(b)

Figure 5. (a) Output characteristics of the HJLTFET and DMGE-HJLTFET at gate-to-source voltage $(\mathrm{Vgs})=1 \mathrm{~V} ;(\mathbf{b})$ output transconductance of the HJLTFET and DMGE-HJLTFET at Vgs $=1 \mathrm{~V}$.

As another important parameter to evaluate the analog performance of the devices, the output transconductance $\left(\mathrm{g}_{\mathrm{ds}}\right)$ can be calculated by the first derivative of the drain current (Ids) with respect to $V_{\mathrm{DS}}$, Equation (2) is the formula of $g_{\mathrm{ds}}$ :

$$
\mathrm{g}_{\mathrm{ds}}=\frac{\mathrm{dI}_{\mathrm{ds}}}{\mathrm{dV} \mathrm{V}_{\mathrm{ds}}}
$$

The comparison of $g_{d s}$ characteristics of the HJLTFET and DMGE-HJLTFET at Vgs $=1 \mathrm{~V}$ is shown in Figure $5 b$. It can be viewed that $g_{d s}$ of the DMGE-HJLTFET was greater than that of HJLTFET within the simulated voltage range. The maximum output conductance of DMGE-HJLTFET was $6.06 \times 10^{-4}$ $\mathrm{S} / \mu \mathrm{m}$ when $\mathrm{Vds}=0.56 \mathrm{~V}$, however, the maximum output conductance of the HJLTFET was $9.46 \times 10^{-5}$ $\mathrm{S} / \mu \mathrm{m}$ when $\mathrm{Vds}=0.38 \mathrm{~V}$. Therefore, the maximum $g_{\mathrm{ds}}$ of the DMGE-HJLTFET was almost seven times more than HJLTFET. 


\subsection{Effect of Device Parameters on the Transfer Characteristics}

Figure 6a shows the impact of source doping concentration on transfer characteristics. As can be seen from this figure, the ON-state current of the DMGE-HJLTFET decreased with the increase of source doping concentration, and it was easy to find that the ON-state current of the DMGE-HJLTFET went from $5.46 \times 10^{-4} \mathrm{~A} / \mu \mathrm{m}$ to $3.61 \times 10^{-4} \mathrm{~A} / \mu \mathrm{m}$ when the source doping concentration increased from $1 \times 10^{17} \mathrm{~cm}^{-3}$ to $1 \times 10^{19} \mathrm{~cm}^{-3}$, the reason for this was that we adopted uniform $\mathrm{N}$-type doping in the source, channel and drain region, and used the charge plasma concept to form a $\mathrm{P}^{+}-\mathrm{I}-\mathrm{N}^{+}$structure with the appropriate workfunction for PG and CG. As a result, the hole concentration formed by polarization in the source region decreased with the increase of the N-type concentration of the lightly doped source. Since the tunnel process was the hole of source valance band to channel conduction band, the ON-state current decreased when we increased the source doping concentration. Moreover, the OFF-state current of DMGE-HJLTFET always remained at the order of $10^{-17} \mathrm{~A} / \mu \mathrm{m}$. Figure $6 \mathrm{~b}$ presents the variation of the ON-state current and the OFF-state current with the increase of source doping concentration, it was very clear that the OFF-state current was minimal when source doping concentration was $5 \times 10^{17} \mathrm{~cm}^{-3}$, as a consequence, the ratio of Ion/Ioff comes up to maximum at this doping concentration as shown in the inserted small graph at Figure $6 \mathrm{~b}$. Therefore, the source doping concentration was selected as $5 \times 10^{17} \mathrm{~cm}^{-3}$ in order to obtain maximal Ion/Ioff value.

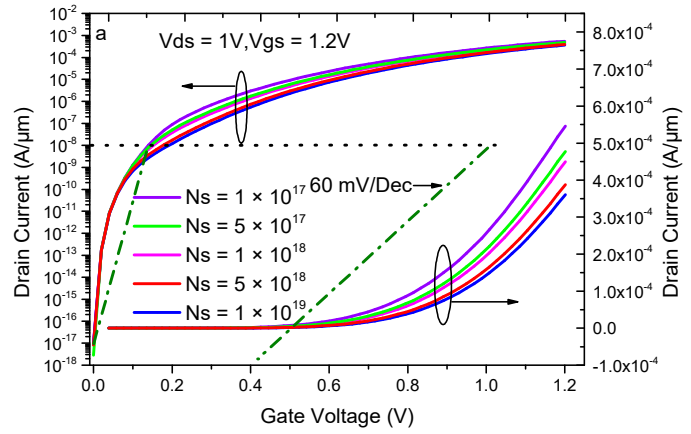

(a)

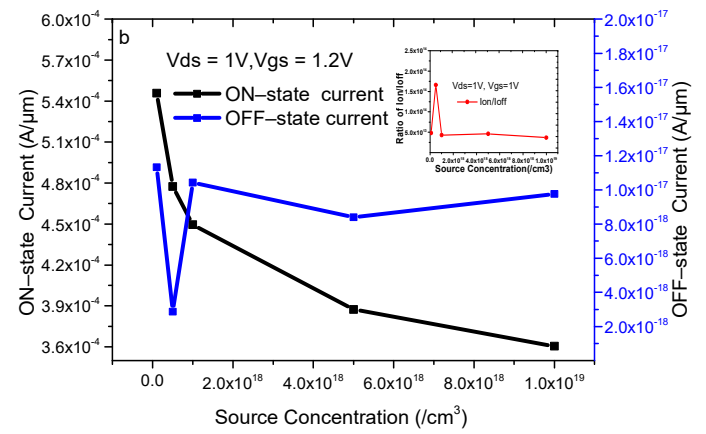

(b)

Figure 6. (a) Transfer characteristics of the DMGE-HJLTFET with different source doping concentration; (b) variation of ON-state current, OFF-state current and Ion/Ioff with different source doping concentration.

We mentioned above that the gate electrode was divided into three parts namely auxiliary gate (M1), control gate (M2) and tunnel gate (M3) with workfunctions $\Phi_{\mathrm{M} 1}, \Phi_{\mathrm{M} 2}$ and $\Phi_{\mathrm{M} 3}$, respectively, where $\Phi_{\mathrm{M} 1}=\Phi_{\mathrm{M} 3}<\Phi_{\mathrm{M} 2}$. Next, the effects of the three parameters on the transfer characteristics will be discussed.

Figure 7a shows the impact of polar gate workfunction $\left(\Phi_{\mathrm{PG}}\right)$ on transfer characteristics with keeping $\Phi_{\mathrm{M} 1}=\Phi_{\mathrm{M} 3}=4.1 \mathrm{eV}$ and keeping $\Phi_{\mathrm{M} 2}=4.4 \mathrm{eV}$. As can be seen clearly from Figure 7a, the ON-state current of the DMGE-HJLTFET increased and the OFF-state current of the DMGE-HJLTFET stayed at the order of $10^{-17} \mathrm{~A} / \mu \mathrm{m}$ with the increase of $\Phi_{\mathrm{PG}}$, so an appropriate choice for $\Phi_{\mathrm{PG}}$ was very important to achieve higher ON-state current and lower OFF-state current simultaneously. The reason for this was that $\Phi_{\mathrm{PG}}$ had a large influence on the polarization charge formation in the source region, the amount of polarization charge increased with the polar gate workfunction, resulting in the variation of energy band, as illustrated in Figure $7 \mathrm{~b}$. Figure $7 \mathrm{~b}$ shows the variation of energy band with different $\Phi_{\mathrm{PG}}$, the position of conduction band and valance band in source region became higher and higher when $\Phi_{\mathrm{PG}}$ went up, which boosted the effective tunneling area of the channel-source interface, and the ON-state current of the DMGE-HJLTFET increased with the increase of $\Phi_{\mathrm{PG}}$. Therefore, Figure $7 \mathrm{~b}$ further demonstrated the conclusion of Figure $7 \mathrm{a}$. 


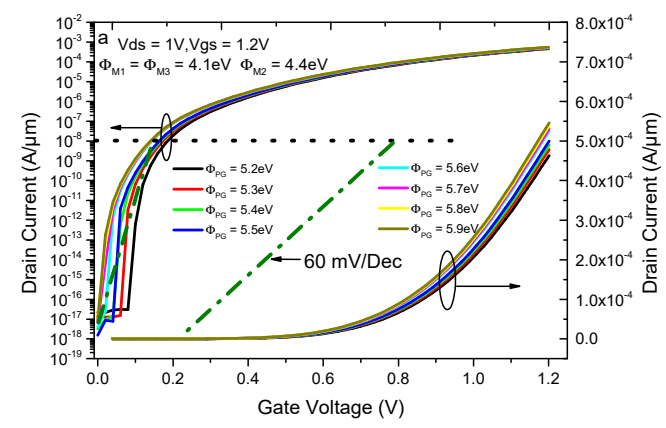

(a)

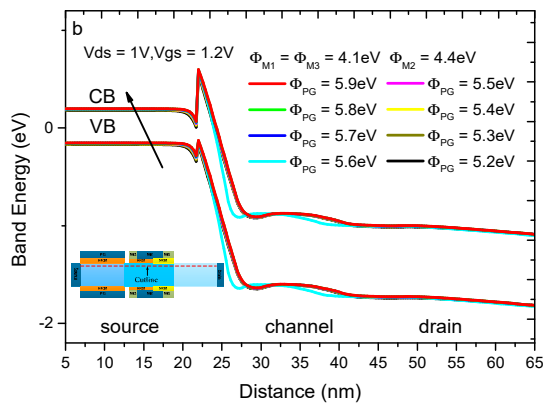

(b)

Figure 7. (a) Transfer characteristics of the DMGE-HJLTFET with different polar gate workfunction $\left(\Phi_{\mathrm{PG}}\right) ;(\mathbf{b})$ the variation of energy band diagram with different polar gate workfunction $\left(\Phi_{\mathrm{PG}}\right)$.

Figure 8a-d indicate the impact of the control gate workfunction $\left(\Phi_{\mathrm{M} 2}\right)$ on transfer characteristics, electric field distribution and band diagram with keeping $\Phi_{\mathrm{M} 1}=\Phi_{\mathrm{M} 3}=4.1 \mathrm{eV}$ and keeping $\Phi_{\mathrm{PG}}=5.9 \mathrm{eV}$. Selection of $\Phi_{\mathrm{M} 2}$ was critical for obtaining higher ratio of Ion/Ioff and prominent SSave, as depicted in Figure 8a. The ON-state current of the DMGE-HJLTFET decreased with the increase of $\Phi_{\mathrm{M} 2}$ and the OFF-state current of the DMGE-HJLTFET decreased with the increase of $\Phi_{\mathrm{M} 2}$ when $\Phi_{\mathrm{M} 2} \leq 4.4 \mathrm{eV}$. In addition, the ambipolar current occurred when $\Phi_{\mathrm{M} 2} \geq 4.5 \mathrm{eV}$ which led to very poor transfer characteristic curves in the DMGE-HJLTFET as shown in Figure 8a. In Figure 8b, it is very clear that the value of electric field under M3 decreased with the increase of $\Phi_{\mathrm{M} 2}$ while the value of the electric field under M1 and M2 decreased with the increase of $\Phi_{\mathrm{M} 2}$ when $\Phi_{\mathrm{M} 2} \leq 4.4 \mathrm{eV}$ and increased with the increase of $\Phi_{\mathrm{M} 2}$ when $\Phi_{\mathrm{M} 2} \geq 4.5 \mathrm{eV}$, which further explains the variation of the ON-state current in Figure $8 \mathrm{a}$ and made the energy band bend severely in this region, as shown in Figure $8 \mathrm{c}, \mathrm{d}$. Figure $8 \mathrm{c}, \mathrm{d}$ depict the energy band diagram in ON-state and OFF-state, where it was observed that the tunneling barrier height increased with the increase of $\Phi_{\mathrm{M} 2}$ and height of barrier in OFF-state was much higher than that of ON-state. Hence, the overall performance of the DMGE-HJLTFET was significantly affected by $\Phi_{\mathrm{M} 2}$, considering all the factors, the optimal value of control gate workfunction was chosen as $4.4 \mathrm{eV}$.

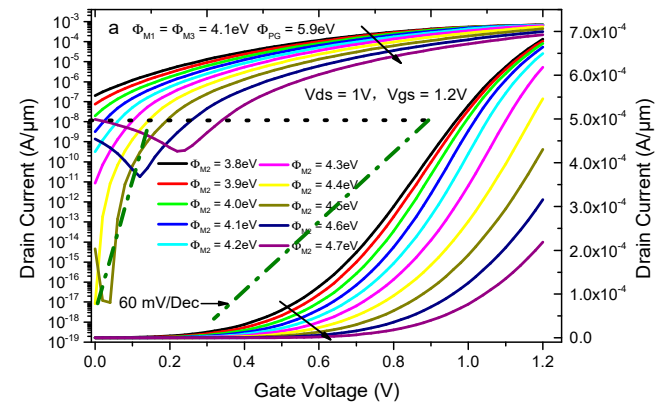

(a)

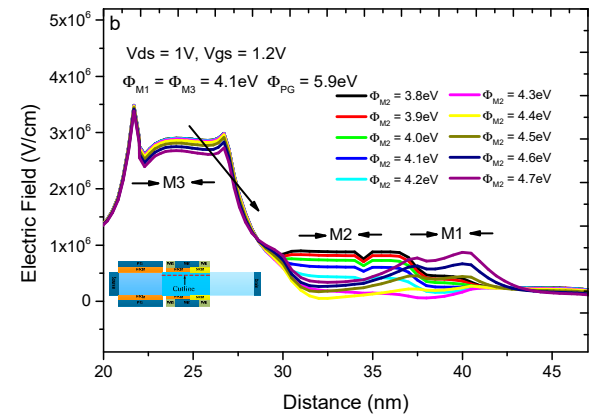

(b)

Figure 8. Cont. 


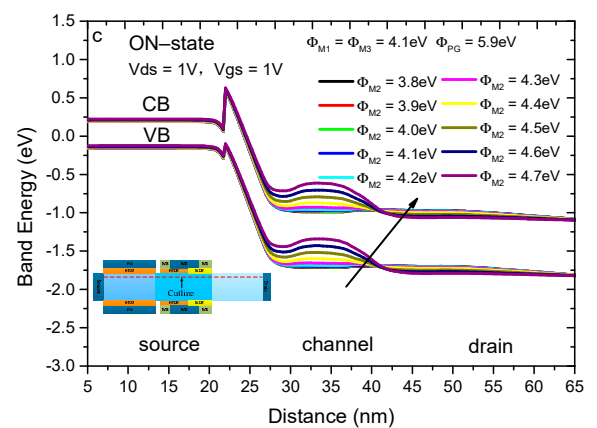

(c)

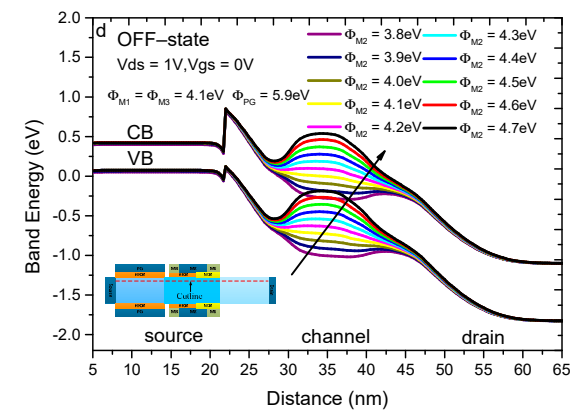

(d)

Figure 8. (a) Transfer characteristics of the DMGE-HJLTFET with different control gate workfunction $\left(\Phi_{\mathrm{M} 2}\right)$; (b) the distribution of electric field with different $\Phi_{\mathrm{M} 2} ;$ (c) the ON-state energy band diagram with different $\Phi_{\mathrm{M} 2} ;$ (d) the OFF-state energy band diagram with different $\Phi_{\mathrm{M} 2}$.

Figure 9a-d illustrate the impact of the auxiliary gate workfunction $\left(\Phi_{\mathrm{M} 1}\right)$ and tunnel gate workfunction $\left(\Phi_{\mathrm{M} 3}\right)$ on transfer characteristics, surface potential, electric field and band diagram with keeping $\Phi_{\mathrm{M} 2}=4.4 \mathrm{eV}$ and keeping $\Phi_{\mathrm{PG}}=5.9 \mathrm{eV}$. The transfer characteristics of the DMGE-HJLTFET at different values of $\Phi_{\mathrm{M} 1}$ is shown in Figure 9a, where $\Phi_{\mathrm{M} 1}$ was varied from $3.7 \mathrm{eV}$ to $4.4 \mathrm{eV}$ in a step of $0.1 \mathrm{eV}$. It could be found through the observation of Figure 9a that the ON-state current and the OFF-state current of the DMGE-HJLTFET decreased with the increase of $\Phi_{\mathrm{M} 1}$, that is to say, appropriate selection of $\Phi_{\mathrm{M} 1}$ was required to achieve higher Ion/Ioff ratio and lower SSave, therefore, $\Phi_{\mathrm{M} 1}=4.1 \mathrm{eV}$ was selected as the optimal value for auxiliary gate workfunction. In fact, the variation of transfer characteristics depended on the changes of surface potential and electric field. Figure $9 b, c$ show the distribution of surface potential and electric field at the device surface. It can be noticed that both of them were markedly influenced by $\Phi_{\mathrm{M} 1}$ and the DMGE-HJLTFET exhibited higher surface potential and electric field at the position of M1 and M3 under small $\Phi_{\mathrm{M} 1}$, in which M1 suppressed the ambipolar leakage current and reduced hot carrier effects (HCEs) by diminishing the lateral electric field at the drain-channel interface. M3 improved the ON-state current by increasing the tunneling probability at the source-channel interface. A higher tunneling rate at the source-channel interface and higher barrier height at drain-channel interface were introduced simultaneously, as shown in Figure 9d. Therefore, $\Phi_{\mathrm{M} 1}$ should be small in terms of ON-state current and OFF-state current, and $\Phi_{\mathrm{M} 1}$ should not be small in terms of barrier height at the drain-channel interface. All things considered, the optimal value of $\Phi_{\mathrm{M} 1}$ was chosen as $4.1 \mathrm{eV}$.

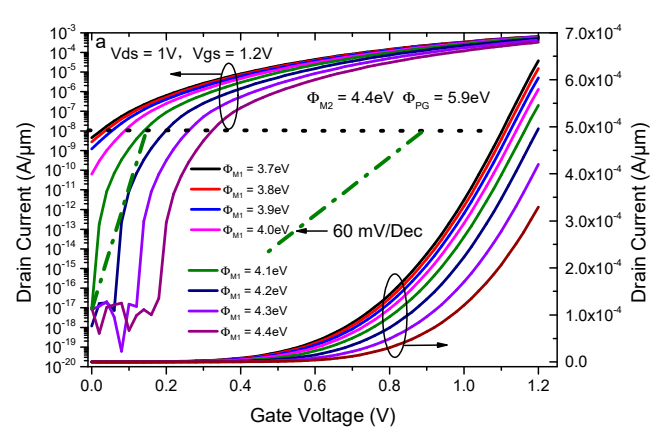

(a)

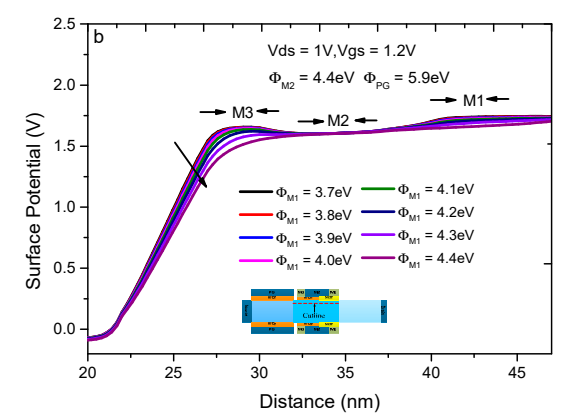

(b)

Figure 9. Cont. 


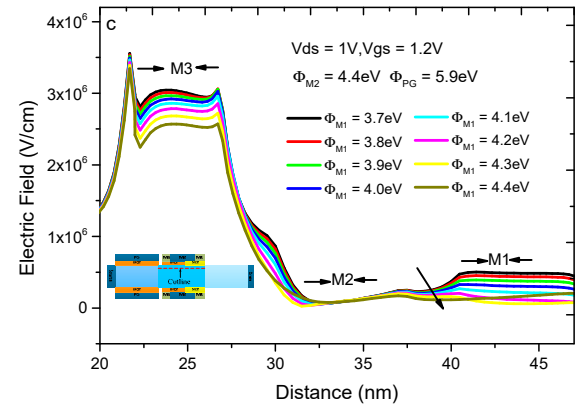

(c)

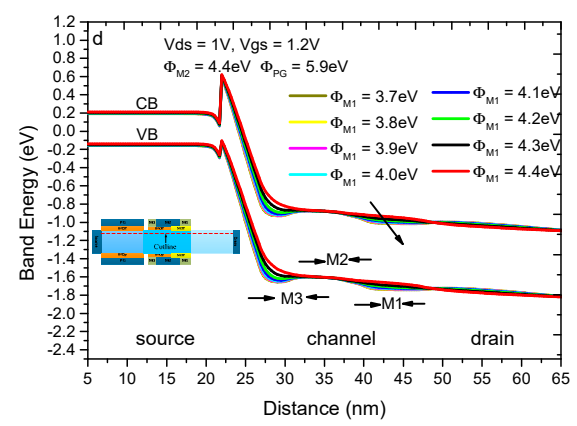

(d)

Figure 9. (a) Transfer characteristics of the DMGE-HJLTFET with different auxillary gate workfunction $\left(\Phi_{\mathrm{M} 1}\right) ;(\mathbf{b})$ the potential distribution with different $\Phi_{\mathrm{M} 1}$; (c) the distribution of electric field with different $\Phi_{\mathrm{M} 1} ;(\mathbf{d})$ the ON-state energy band diagram with different $\Phi_{\mathrm{M} 1}$.

\subsection{Comparison in Terms of Analog/RF Performance}

As we know, it is very necessary to research the frequency characteristics of the integrated circuits, which is profoundly affected by parasitic capacitances of devices. Apparently, the characteristics of Cgg (gate capacitance), Cgs (capacitance of gate to source) and Cgd (capacitance of gate to drain) is of great significance to evaluate the frequency characteristics and analog application ability of devices [38]. Figure 10a shows the capacitance of the HJLTFET versus Vgs and Figure 10b shows the capacitance of the DMGE-HJLTFET versus Vgs. As can be observed from Figure 10a,b, the trend of capacitance curve versus Vgs was similar for the HJLTFET and DMGE-HJLTFET, Cgg and Cgd both remained at a small value when Vgs $<$ Vds and increased rapidly with the increasing Vgs when Vgs $>$ Vds, and Cgs maintained a very small value at this condition. In addition, the curve of Cgg and Cgd was highly adjacent due to the existence of a polar gate which separated the control gate electrode far from the source electrode, as a result, coupling of the control gate electrode and source electrode was relatively slight, so, Cgg was mainly determined by Cgd.

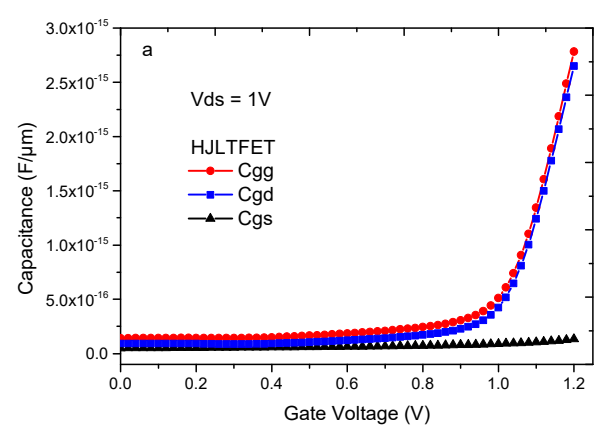

(a)

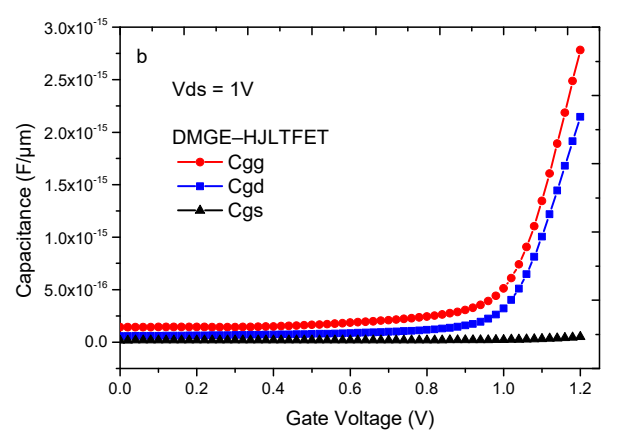

(b)

Figure 10. (a) Capacitance of HJLTFET versus Vgs; (b) capacitance of DMGE-HJLTFET versus Vgs.

The cut-off frequency $\left(f_{T}\right)$ and gain bandwidth (GBW) are important indicators for evaluating RF performance of devices. Based on Figure 10a,b, the cut-off frequency $\left(f_{T}\right)$ and the gain bandwidth (GBW) of the HJLTFET and DMGE- HJLTFET are discussed in next section.

As shown in Equation (3), cut-off frequency $\left(f_{T}\right)$ can be expressed as ratio of gm to $C_{g g}$ :

$$
f_{T}=\frac{g_{m}}{2 \pi C_{g s} \sqrt{1+2 \frac{C_{g d}}{C_{g s}}}} \approx \frac{g_{m}}{2 \pi\left(C_{g s}+C_{g d}\right)}=\frac{g_{m}}{2 \pi C_{g g}} .
$$


The GBW can be expressed as a ratio of gm to Cgd for the DC gain value equal to 10 , as shown in Equation (4) [39,40]:

$$
\mathrm{GBW}=\frac{\mathrm{g}_{\mathrm{m}}}{2 \pi 10 \mathrm{C}_{\mathrm{gd}}} .
$$

Figure 11a,b respectively show the characteristic curves of $f_{T}$ and GBW of the HJLTFET and DMGE-HJLTFET. Benefitting from a large transconductance induced by a lightly doped source and gate-engineered heterostructure, the DMGE-HJLTFET could achieve a maximum $f_{T}$ of $423 \mathrm{GHz}$ at $\mathrm{Vgs}=0.92 \mathrm{~V}$ and a maximum GBW of $82 \mathrm{GHz}$ at $\mathrm{Vgs}=0.88 \mathrm{~V}$, respectively. However, the maximum $\mathrm{f}_{\mathrm{T}}$ of the HJLTFET was only $63 \mathrm{GHz}$ at $\mathrm{Vgs}=0.98 \mathrm{~V}$ and maximum GBW of the HJLTFET was only $72 \mathrm{GHz}$ at $\mathrm{Vgs}=1.2 \mathrm{~V}$. Therefore, the DMGE-HJLTFET could be used in future low power and high frequency applications.

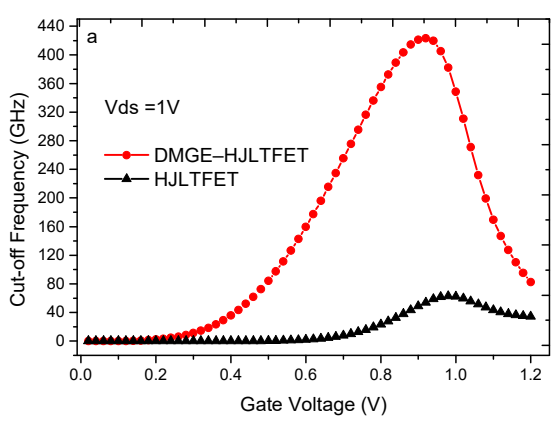

(a)

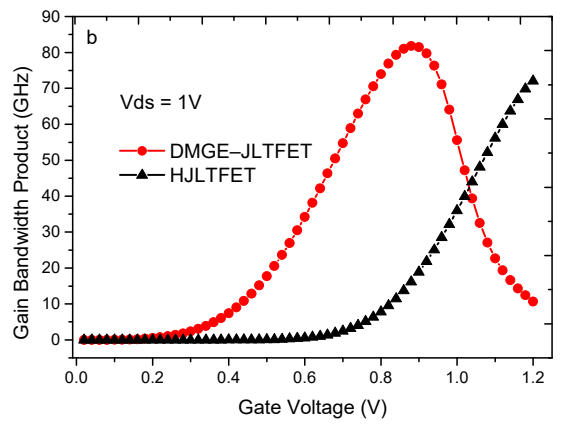

(b)

Figure 11. (a) The cut-off frequency $\left(f_{T}\right)$ characteristic curves of the HJLTFET and DMGE-HJLTFET; (b) the gain bandwidth (GBW) characteristic curves of HJLTFET and DMGE-HJLTFET.

\section{Conclusions}

In this paper, a novel structure of a dual material gate-engineered heterostructure junctionless tunnel field-effect transistor (DMGE-HJLTFET) with a lightly doped source was constructed and researched to improve the performance of conventional InAs/ $\mathrm{GaAs}_{0.1} \mathrm{Sb}_{0.9}$ HJLTFET. Similar to the conventional HJLTFET, the DMGE-HJLTFET still adopted a InAs/ $\mathrm{GaAs}_{0.1} \mathrm{Sb}_{0.9}$ heterojunction at the source and channel interface and employed a polarization electric field at the arsenic heterojunction induced by the lattice mismatch to improve band to band tunneling (BTBT) current. In addition, based on the gate-engineered concept, the gate electrode of DMGE-HJLTFET was divided into three parts to improve the ON-state current and suppress the OFF-state current simultaneously. In this regard, a lightly doped source was used to further optimize the ON-state current and the OFF-state current of this device. In this simulation, the effects of the source doping concentration, polar gate workfunction, control gate workfunction, tunnel gate workfunction and auxiliary gate workfunction on the performance of the DMGE-HJLTFET were researched systematically to optimize the overall device performance. Simulation results indicated that the DMGE-HJLTFET provided superior metrics in terms of logic and analog/RF performance as compared with the conventional HJLTFET, the maximum ON-state current and transconductance of the DMGE-HJLTFET increased up to $5.46 \times 10^{-4} \mathrm{~A} / \mu \mathrm{m}$ and $1.51 \times 10^{-3} \mathrm{~S} / \mu \mathrm{m}$ at Vds $=1 \mathrm{~V}$. Moreover, SSave of the DMGE-HJLTFET was as low as $15.4 \mathrm{mV} / \mathrm{Dec}$ at low drain voltages. Also, the DMGE-HJLTFET could achieve a maximum $f_{\mathrm{T}}$ of $423 \mathrm{GHz}$ at $\mathrm{Vgs}=0.92 \mathrm{~V}$ and a maximum GBW of $82 \mathrm{GHz}$ at $\mathrm{Vgs}=0.88 \mathrm{~V}$, respectively. Therefore, the DMGE-HJLTFET could be used in future low power and high frequency applications.

Author Contributions: Conceptualization, H.L., T.H., W.L., S.C., S.W. and H.X.; methodology, H.X. and T.H.; software, H.X. and W.L.; validation, H.L. and H.X.; formal analysis, H.X.; investigation, H.X.; resources, H.X.; data curation, H.X.; writing-original draft preparation, H.X.; writing-review and editing, H.X.; visualization, H.X.; supervision, H.X.; project administration, H.L.; funding acquisition, H.L. 
Funding: This research was funded by National Natural Science Foundation of China (Grant No. U1866212) and in part by Foundation for Fundamental Research of China (Grant No. JSZL2016110B003) and Major Fundamental Research Program of Shaanxi (Grant No. 2017ZDJC-26) and Innovation Foundation of Radiation Application (Grant No. KFZC2018040206).

Conflicts of Interest: The authors declare no conflict of interest.

\section{References}

1. Choi, W.Y.; Park, B.-G.; Lee, J.D.; Liu, T.-J.K. Tunneling Field-Effect Transistors (TFETs) With Subthreshold Swing (SS) Less Than $60 \mathrm{mV} / \mathrm{dec}$. IEEE Electron. Device Lett. 2007, 28, 743-745. [CrossRef]

2. Anghel, C.; Gupta, A.; Amara, A.; Vladimirescu, A. 30-nm Tunnel FET with Improved Performance and Reduced Ambipolar Current. IEEE Trans. Electron. Devices 2011, 58, 1649-1654. [CrossRef]

3. Kim, J.H.; Kim, S.; Park, B.-G. Double-Gate TFET with Vertical Channel Sandwiched by Lightly Doped Si. IEEE Trans. Electron. Devices 2019, 66, 1656-1661. [CrossRef]

4. Shaikh, M.R.U.; Loan, S.A. Drain-Engineered TFET with Fully Suppressed Ambipolarity for High-Frequency Application. IEEE Trans. Electron. Devices 2019, 66, 1628-1634. [CrossRef]

5. Yang, Z. Tunnel Field-Effect Transistor with an L-Shaped Gate. IEEE Electron. Device Lett. 2016, $37,1$. [CrossRef]

6. Kim, S.W.; Kim, J.H.; Liu, Ts.K.; Choi, W.Y.; Park, B.-G. Demonstration of L-Shaped Tunnel Field-Effect Transistors. IEEE Trans. Electron. Devices 2016, 63, 1. [CrossRef]

7. Faraz, N.; Seop, Y.Y. Impact of Quantum Confinement on Band-to-Band Tunneling of Line-Tunneling Type L-Shaped Tunnel Field-Effect Transistor. IEEE Trans. Electron. Devices 2019, 66, 2010-2016.

8. Kim, J.H.; Kim, S.; Park, B.-G. Symmetric U-Shaped Gate Tunnel Field-Effect Transistor. IEEE Trans. Electron. Devices 2017, 64, 1343-1349.

9. Wang, W.; Wang, Pe.; Zhang, Ch.; Lin, X.; Liu, Xi.; Sun, Qi.; Zhou, P.; Zhang, D.W. Design of U-Shape Channel Tunnel FETs with SiGe Source Regions. IEEE Trans. Electron. Devices 2014, 61, 193-197. [CrossRef]

10. Nam, H.; Cho, M.H.; Shin, C. Symmetric tunnel field-effect transistor (S-TFET). Curr. Appl. Phys. 2015, 15, 71-77. [CrossRef]

11. Lee, H.; Park, S.; Lee, Y.; Nam, H.; Shin, C. Random variation analysis and variation-aware design of symmetric tunnel field-effect transistor. IEEE Trans. Electron. Devices 2015, 62, 1778-1783.

12. Jiang, Z.; Zhuang, Y.; Li, C.; Wang, P. Dual Sources U-shape Gate Tunnel FETs with High On-current and Steep SS. In Proceedings of the 2016 16th International Workshop on Junction Technology (IWJT), Shanghai, China, 9-10 May 2015; pp. 25-27.

13. Sola, W.; Sangsig, K. Covered Source-Channel Tunnel Field-Effect Transistors with Trench Gate Structures. IEEE Trans. Nanotechnol. 2018, 18, 114-118.

14. Li, W.; Liu, H.; Wang, S.; Chen, S.; Yang, Z. Design of High Performance Si/SiGe Heterojunction Tunneling FETs with a T-Shaped Gate. Nanoscale Res. Lett. 2017, 12, 198. [CrossRef] [PubMed]

15. Szabo, A.; Koester, S.J.; Luisier, M. Ab-Initio Simulation of van der Waals $\mathrm{MoTe}_{2}-\mathrm{SnS}_{2}$. Heterotunneling FETs for Low-Power Electronics. IEEE Electron. Device Lett. 2015, 36, 514-516. [CrossRef]

16. Cao, J.; Logoteta, D.; Özkaya, S.; Biel, B.; Cresti, A.; Pala, M.G.; Esseni, D. Operation and Design of van der Waals Tunnel Transistors: A 3-D Quantum Transport Study. IEEE Trans. Electron. Devices 2016, 63, 4388-4394. [CrossRef]

17. Raad, B.R.; Tirkey, S.; Sharma, D.; Kondekar, P. A New Design Approach of Dopingless Tunnel FET for Enhancement of Device Characteristics. IEEE Trans. Electron. Devices 2017, 64, 1830-1836. [CrossRef]

18. Singh, D.; Pandey, S.; Nigam, K.; Sharma, D.; Yadav, D.S.; Kondekar, P. A Charge-Plasma-Based Dielectric-Modulated Junctionless TFET for Biosensor Label-Free Detection. IEEE Trans. Electron. Devices 2017, 64, 271-277. [CrossRef]

19. Kumar, M.J.; Janardhanan, S. Doping-Less Tunnel Field Effect Transistor: Design and Investigation. IEEE Trans. Electron. Devices 2013, 60, 3285-3290. [CrossRef]

20. Aslam, M.; Sharma, D.; Yadav, S.; Soni, D.; Sharma, N.; Gedam, A. A comparative investigation of low work-function metal implantation in the oxide region for improving electrostatic characteristics of charge plasma TFET. Micro Nano Lett. 2019, 14, 123-128. [CrossRef] 
21. Ameen, T.A.; Ilatikhameneh, H.; Fay, P.; Seabaugh, A.; Rahman, R.; Klimeck, G. Alloy Engineered Nitride Tunneling Field Effect Transistor: A Solution for the Challenge of Heterojunction TFETs. IEEE Trans. Electron. Devices 2019, 66, 736-742. [CrossRef]

22. Hoda, T.S.; Saleh, G.S.; Yousefi, R.; Aderang, H. A computational study of a carbon nanotube junctionless tunneling field-effect transistor (CNT-JLTFET) based on the charge plasma concept. Superlattices Microstruct. 2019, 125, 168-176.

23. Ghosh, B.; Bal, P.; Mondal, P. A junctionless tunnel field effect transistor with low subthreshold slope. J. Comput. Electron. 2013, 12, 428-436. [CrossRef]

24. Aghandeh, H.; Ziabari, S.A.S. Gate engineered heterostructure junctionless TFET with Gaussian doping profile for ambipolar suppression and electrical performance improvement. Superlattices Microstruct. 2017, 111, 103-134. [CrossRef]

25. Abadi, R.M.I.; Ziabari, S.A.S. Improved performance of nanoscale junctionless tunnel field-effect transistor based on gate engineering approach. Appl. Phys. A 2016, 122, 988. [CrossRef]

26. Rahimian, M.; Fathipour, M. Improvement of electrical performance in junctionless nanowire TFET using hetero-gate-dielectric. Mater. Sci. Semicond. Process. 2017, 63, 142-152. [CrossRef]

27. Tirkey, S.; Nigam, K.; Pandey, S.; Sharma, D.; Kondekar, P. Investigation of gate material engineering in junctionless TFET to overcome the trade-off between ambipolarity and RF/linearity metrics. Superlattices Microstruct. 2017, 109, 307-315.

28. Basak, S.; Asthana, P.K.; Goswami, Y. Leakage current reduction in junctionless tunnel FET using a lightly. doped source. Appl. Phys. 2015, 118, 1527-1533. [CrossRef]

29. Bal, P.; Akram, M.W.; Mondal, P.; Ghosh, B. Performance estimation of sub-30 nm junctionless tunnel FET (JLTFET). J. Comput. Electron. 2013, 12, 782-789. [CrossRef]

30. Lee, J.C.; Ahn, T.J.; Yu, Y.S. Si/Ge Hetero Tunnel Field-Effect Transistor with Junctionless Channel Based on Nanowire. J. Nanosci. Nanotechnol. 2019, 19, 6750-6754. [CrossRef]

31. Leung, G.; Chui, C.O. Variability Impact of Random Dopant Fluctuation on Nanoscale Junctionless FinFETs. IEEE Electron. Device Lett. 2012, 33, 767-769. [CrossRef]

32. Ahangari, Z. Design and analysis of energy efficient semi-junctionless $n+n+p$ heterojunction $p$-channel tunnel field effect transistor. Mater. Res. Express 2019, 6, 6750-6754. [CrossRef]

33. Vanlalawmpuia, K.; Rajesh, S.; Brinda, B. Performance evaluation of hetero-stacked TFET for variation in lateral straggle and its application as digital inverter. Appl. Phys. A Mater. Sci. Process. 2018, 124, 1-8. [CrossRef]

34. Biswas, A.; Luong, G.V.; Chowdhury, M.F.; Alper, C.; Zhao, Qi.; Udrea, F. Benchmarking of Homojunction Strained-Si NW Tunnel FETs for Basic Analog Functions. IEEE Trans. Electron. Devices 2017, 64, 1441-1448. [CrossRef]

35. Chakraborty, A.; Sarkar, A. Investigation of analog/RF performance of staggered heterojunctions based nanowire tunneling field-effect transistors. Superlattices Microstruct. 2015, 80, 125-135. [CrossRef]

36. Biswal, S.M.; Baral, B.; De, D.; Sarkar, A. Simulation and comparative study on analog/RF and linearity performance of III-V semiconductor-based staggered heterojunction and InAs nanowire(nw) Tunnel FET. Superlattices Microstruct. 2019, 25, 1855-1861. [CrossRef]

37. Kr, M.S.; Brinda, B. Impact of interface traps on performance of Gate-on-Source/Channel SOI TFET. Microelectron. Reliab. 2019, 94,1-12.

38. Sarkar, A.; Sarkar, C.K. RF and analogue performance investigation of DG tunnel FET. Electron. Lett. 2010, 1, 210-217. [CrossRef]

39. Chen, S.; Liu, H.; Wang, S.; Li, W.; Wang, X.; Zhao, L. Analog/RF Performance of T-Shape Gate Dual-Source Tunnel Field-Effect Transistor. Nanoscale Res. Lett. 2018, 13, 321. [CrossRef]

40. Narwal, S.; Chauhan, S.S. Investigation of RF and linearity performance of electrode work-function engineered HDB vertical TFET. Micro Nano Lett. 2019, 14, 17-21. [CrossRef]

(C) 2019 by the authors. Licensee MDPI, Basel, Switzerland. This article is an open access article distributed under the terms and conditions of the Creative Commons Attribution (CC BY) license (http://creativecommons.org/licenses/by/4.0/). 